\title{
De-Excitation Gamma-ray Line Emission from the Galactic Center
}

\author{
D.O. Chernyshov*1,2,4,5, V.A. Dogiel ${ }^{2}$, V.Tatischeff ${ }^{3}$, K.-S. Cheng ${ }^{1}$, , C.-M. Ko ${ }^{5}$, and \\ W.-H. Ip 5 \\ ${ }^{1}$ Department of Physics, University of Hong Kong, Pokfulam Road, Hong Kong, China \\ ${ }^{2}$ I.E.Tamm Theoretical Physics Division of P.N.Lebedev Institute, Leninskii pr, 53, 119991 \\ Moscow, Russia \\ ${ }^{3}$ Centre de Spectrometrie Nucleaire et de Spectrometrie de Masse, IN2P3-CNRS and Universite \\ Paris-Sud, F-91405 Orsay Campus, France \\ ${ }^{4}$ Moscow Institute of Physics and Technology, Institutskii lane, 141700 Moscow Region, \\ Dolgoprudnii, Russia \\ ${ }^{5}$ Institute of Astronomy, National Central University, Jhongli 320, Taiwan
}

\begin{abstract}
A future detection of de-excitation gamma-ray lines from the Galactic center region would provide unique information on the high-energy processes induced by the the central black hole and the physical conditions of the emitting region.

We analyse the intensity of nuclear de-excitation lines in the direction of the Galactic center produced by subrelativistic protons, which are generated by star capture by the central black hole. With the metallicity two times higher than the solar one the total flux in gamma-ray lines of energies below $8 \mathrm{MeV}$ is about $10^{-4} \mathrm{~cm}^{-2} \mathrm{~s}^{-1}$. The most promising lines for detection are those at 4.44 and $\sim 6.2 \mathrm{MeV}$, with a predicted flux in each line of $\approx 10^{-5}$ photons $\mathrm{cm}^{-2} \mathrm{~s}^{-1}$.

We also analyze the possibility of detection of these lines by INTEGRAL and future missions.
\end{abstract}

The Extreme Sky: Sampling the Universe above $10 \mathrm{keV}$

October 13-17 2009

Otranto, Italy

\footnotetext{
* Speaker.
} 


\section{Introduction}

As observations show, there is a supermassive black hole $\left(\mathrm{Sgr} \mathrm{A}^{*}\right)$ in the center of our Galaxy with a mass of $(4.31 \pm 0.06) \times 10^{6} M_{\odot}$ [7]. ASCA, Chandra and Suzaku found emission of 6.5 $\mathrm{keV}$ plasma from the central $\sim 1^{\circ}$-radius region which cannot be heated by SNe. The required energy input is $\sim 10^{41} \mathrm{erg} / \mathrm{s}[9,14]$. Suzaku observed a flux of nonthermal X-ray emission from the Galactic center. The total flux in the range $14-40 \mathrm{keV}$ is $4 \times 10^{36} \mathrm{erg} / \mathrm{s}$ [22]. The molecular gas in the Galactic buldge is much warmer $(\mathrm{T} \sim 100 \mathrm{~K})$ than in other part of the Galactic disk ( $\mathrm{T}$ $\sim 20 \mathrm{~K})$. No evident sources of heating are observed there. This heating may be associated with a high density of subrelativistic cosmic rays near the Galactic center [23] which can produce also the observed $6.4 \mathrm{keV}$ flux from molecular clouds. It is likely that all these processes can be somehow connected to accretion processes on central black hole.

However, attempts to find a strong X-ray source in the Galactic Center (GC) have failed. The Chandra X-ray Observatory resolved only a weak X-ray point source at the position of Sgr $\mathrm{A}^{*}$ with a flux $L_{X} \sim 10^{33} \mathrm{erg} \mathrm{s}^{-1}$ [3], though moderate X-ray flares were observed by Chandra [16]. As it was mentioned by [9] and [14] this "X-ray quiet" Sgr A* is in sharp contrast to the high X-ray activity of the surrounding diffuse hot plasmas. One likely scenario is that the Galactic nucleus was brighter in the past, possibly caused by a surge accretion onto the massive back hole [19].

Massive black holes (MBH) at galactic centers are sources of high energetic activity. X-ray observations of these sources revealed flares of hard X-ray photons releasing a maximum power of $10^{44} \mathrm{erg} \mathrm{s}^{-1}$. These flares are supposed to be due to processes of accretion and tidal disruption of stars by the massive black holes [18].

The capture radius of a black hole - the maximal distance from the MBH where the tidal forces can overwhelm the stellar self-gravity and tear the star apart - is given by

$$
R_{T} \approx 1.4 \times 10^{13} M_{6}^{1 / 3} m_{*}^{-1 / 3} r_{*} \mathrm{~cm}
$$

where $m_{*}=M_{*} / M_{\odot}, M_{6}=M_{\mathrm{bh}} / 10^{6} M_{\odot}, r_{*}=R_{*} / R_{\odot}, M_{*}$ and $R_{*}$ are the star's mass and radius, $M_{\mathrm{bh}}$ the mass of the black hole, and $M_{\odot}$ and $R_{\odot}$ are the solar mass and radius. When a star comes within the capture radius of a black hole, the tidal force produced by the black hole is strong enough to capture the star $[17,15]$. If the mass of the black hole is higher than $\sim 10^{8} \mathrm{M}_{\odot}$, the star directly falls into the black hole event horizon and not much interesting phenomena can be observed. However, if the mass of the black hole is less than $\sim 10^{8} \mathrm{M}_{\odot}$, the star is torn apart. Roughly half of the star is captured by the black hole and the disrupted debris form in a few months a circular transient accretion disk around the black hole [21].

A total tidal disruption of a star occurs when the penetration parameter $b^{-1} \gg 1$, where $b$ is the ratio of $r_{p}$ - the periapse distance (distance of closest approach) to the tidal radius $R_{T}$. The tidal disruption rate can be approximated to within an order of magnitude from an analysis of star dynamics near a black hole via the Fokker-Planck equation. For the parameters of the GC it gives the rate $v_{s} \sim 10^{-4}$ years $^{-1}$ [1], which is in agreement with more detailed calculations of [20] who obtained $v_{s} \sim 5 \times 10^{-5}$ years $^{-1}$. Tidal disruption processes were perhaps already observed in cosmological galaxy surveys [6].

Below we examine a model of de-excitation line emission, assuming nuclear interactions of subrelativistic protons generated by processes of star disruption at the GC. 


\section{Production and propagation of subrelativistic protons}

Once passing the pericenter, the star is tidally disrupted into a very long and dilute gas stream. The outcome of tidal disruption is that some energy is extracted out of the orbit to unbind the star and accelerate the debris. Initially about $50 \%$ of the stellar mass becomes tightly bound to the black hole, while the remainder $50 \%$ of the stellar mass is forcefully ejected [2]. The kinetic energy carried by the ejected debris is a function of the penetration parameter $b$ and can significantly exceed that released by a normal supernova $\left(\sim 10^{51} \mathrm{erg}\right)$ if the orbit is highly penetrating [1],

$$
W \sim 4 \times 10^{52}\left(\frac{M_{*}}{M_{\odot}}\right)^{2}\left(\frac{R_{*}}{R_{\odot}}\right)^{-1}\left(\frac{M_{\mathrm{bh}} / M_{*}}{10^{6}}\right)^{1 / 3}\left(\frac{b}{0.1}\right)^{-2} \text { erg. }
$$

Thus average kinetic energy per baryon is given by

$$
E_{\text {esc }} \sim 42 \eta\left(\frac{M_{*}}{M_{\odot}}\right)\left(\frac{R_{*}}{R_{\odot}}\right)^{-1}\left(\frac{M_{\mathrm{bh}} / M_{*}}{10^{6}}\right)^{1 / 3}\left(\frac{b}{0.1}\right)^{-2} \mathrm{MeV} .
$$

Here the parameter $\eta$ is the ratio of $50 \%$ mass of the star to escaping mass of the star. For the blackhole mass $M_{\mathrm{bh}}=4.31 \times 10^{6} M_{\odot}$ the energy of escaping particles is $E_{\mathrm{esc}} \sim 68 \eta(b / 0.1)^{-2} \mathrm{MeV}$ nucleon $^{-1}$ when a one-solar mass star is captured. The parameter $b=r_{p} / R_{T}$ where $r_{p}$ is radius of pereastrum is unknown. However $b$ should be less than unity for star to be disrupted. In our work instead of choosing right value of $b$ we will use the convenient value of $E_{\text {esc }}$ in range $10-100 \mathrm{MeV}$.

Below the energy distribution of these erupted nuclei is taken as a simple Gaussian distribution

$$
Q_{0}^{\mathrm{esc}}=\frac{N}{\sigma \sqrt{2 \pi}} \exp \left[-\frac{\left(E-E_{\mathrm{esc}}\right)^{2}}{2 \sigma^{2}}\right],
$$

where $N$ is total amount of particles ejected by one stellar capture. Results of calculations for different values of $\sigma$ do not differ much from each other as long as $\sigma<E_{\text {esc }}$.

For a single capture of a one-solar mass star the total number of unbounded particles is $N \leq$ $10^{57}$ and the total kinetic energy in these particles is $W \leq 10^{53} \mathrm{erg}$. For the star capture frequency $v_{s} \simeq 10^{-5}-10^{-4}$ year $^{-1}$ it gives a power input $\dot{W} \leq 3 \times 10^{41} \mathrm{erg} \mathrm{s}^{-1}$.

Details of the proton spatial distribution in the GC region, as well as the mechanism of proton propagation are inessential for calculations of the total gamma-ray line flux. We use, nevertheless, kinetic equations in which processes of propagation are included, and then integrate the obtained solution over the volume of emission. The effect of propagation reduces in this case to a proper estimate of the proton flux leaving the emission region.

Propagation of cosmic rays in the Galaxy is described as a diffusion phenomenon, with a phenomenological diffusion coefficient whose value and energy dependence is derived from observational data, e.g. from the chemical composition of cosmic rays measured near Earth. This method leads to a diffusion coefficient of about $10^{27} \mathrm{~cm}^{2} \mathrm{~s}^{-1}$ [4]. Processes of particle propagation in the GC region are questionable since we do not know much about the physical conditions of the ambient medium. Below we provide our calculations for the diffusion coefficient $D=10^{27}$ $\mathrm{cm}^{2} \mathrm{~s}^{-1}$, though, in principle, its value may be a function of particle energy (as well as of spatial coordinates and even time). We suppose, however, that this simplification is acceptable because the energy range of relevance for gamma-ray line production is quite narrow. 
Another important parameter of the model is the mean density of the medium into which the fast nuclei propagate. Recent observations with the satellites ASCA, Chandra and Suzaku showed that the $1-2^{\circ}$ radius central region is filled with a hot gas of temperature $6-10 \mathrm{keV}$ and density $n \simeq 0.1-0.4 \mathrm{~cm}^{-3}[9,14,10]$. In our calculations we use the average value $n=0.2 \mathrm{~cm}^{-2}$.

The time-dependent spectrum of subrelativistic protons erupted from the central region can be calculated from the equation

$$
\frac{\partial f}{\partial t}-\nabla(D \nabla f)+\frac{\partial}{\partial E}\left(\frac{d E}{d t} f\right)=Q(E, t)
$$

where $d E / d t \equiv b(E)$ is the rate of proton energy losses. Subrelativistic protons lose their energy by Coulomb collisions. The rate of losses for these protons can be presented in the form

$$
b(E)=-\frac{2 \pi n e^{4}}{m_{e} \mathrm{~V}} \ln \Lambda \simeq-\frac{a}{\sqrt{E}} .
$$

The injection of protons by processes of star capture can be described by

$$
Q(E, \mathbf{r}, t)=\sum_{k=0} Q_{k}(E) \delta\left(t-t_{k}\right) \delta(\mathbf{r}),
$$

where $t_{k}=k \times T$ is the injection time and the functions $Q_{k}(E)$ are given by Eq. (2.3). The mean time of star capture by the massive black hole in the Galaxy is taken to be $T \simeq 10^{4}$ years.

\section{Gamma-ray line emission from the Galactic center region}

Collisions of subrelativistic nuclei with ambient matter can lead to nuclear excitation and result in emission of de-excitation gamma-ray lines. These lines may be a good tracer for subrelativistic cosmic rays, because the line brightness can give us information about the amount of subrelativistic particles.

According to the present model the origin of subrelativistic cosmic rays near the GC is different than in other part of the galactic disk, a positive detection of de-excitation gamma-ray lines would provide important information about star accretion processes at the GC.

We calculate the gamma-ray flux emitted at time $t=T$ after a star capture in a given nuclear de-excitation line from

$$
F_{\gamma}(t)=\frac{n_{H}}{4 \pi D^{2}} \sum_{i j} \frac{n_{j}}{n_{H}} \int_{0}^{\infty} F_{i}\left(E_{i}, t\right) v_{i}\left(E_{i}\right) \sigma_{i j}\left(E_{i}\right) d E_{i}
$$

where $i$ and $j$ range over the accelerated and ambient particle species that contribute to the production of the gamma-ray line considered, $n_{\mathrm{H}}=0.2 \mathrm{~cm}^{-3}$ is the adopted mean density of $\mathrm{H}$ atoms in the Galactic center region, $n_{j}$ is the density of the ambient constituent $j, F_{i}\left(E_{i}, t\right)$ is the energy spectrum at time $t$ of the fast particles in the interaction region (Eq. 15), $v_{i}$ is the velocity of these particles, $\sigma_{i j}$ is the cross section for the reaction of interest between species $i$ and $j$, and $D=8 \mathrm{kpc}$ is the distance to the Galactic center [8]. To take into account the measured enhancement of the metal abundances in the Galactic center region [5], we assume the abundances of ambient $\mathrm{C}$ and heavier elements to be twice solar [13]. This is consistent with the recent estimate from Suzaku 

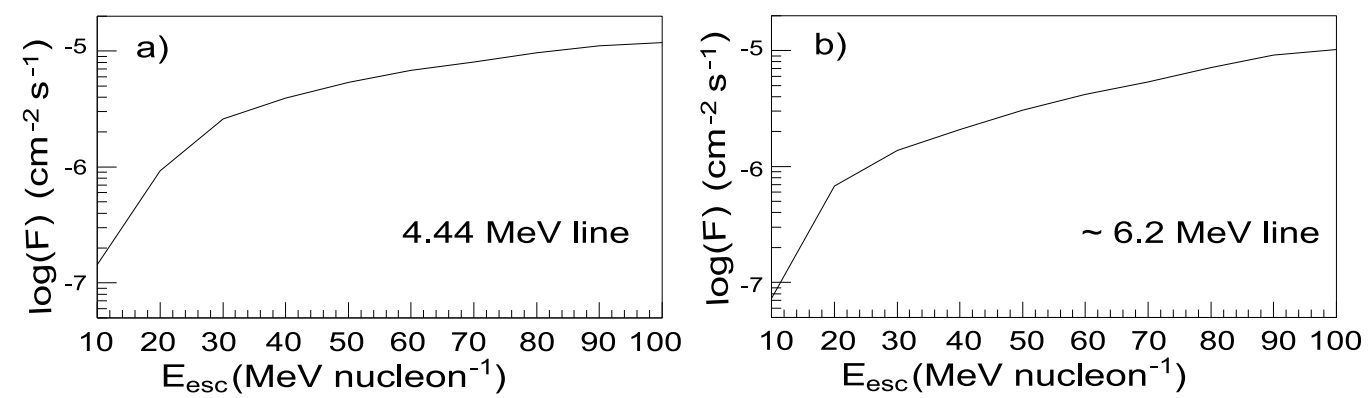

Figure 1: Predicted fluxes in (a) the $4.44 \mathrm{MeV}$ line and (b) the $\sim 6.2 \mathrm{MeV}$ line complex as a function of $E_{\text {esc }}$.

observations of [11], who found the Fe abundance to be between 1.5 and 2.3 times solar. In the case of a low-mass star capture, we expect the line emission to be mainly due to proton and $\alpha$ particle reactions with ambient heavy nuclei. We took into the $\alpha$ particles assuming, for simplicity, $F_{\alpha}(E, t)=X_{\alpha} F_{p}(E, t)$, where the energy $E$ is expressed in $\mathrm{MeV}$ nucleon $^{-1}$ and $X_{\alpha}=0.1$ is the accelerated $\alpha / p$ abundance ratio.

Figure 1 shows calculated fluxes in the 4.44 and $\sim 6.2 \mathrm{MeV}$ lines as a function of $E_{\text {esc }}$. These two lines are mainly produced by proton and $\alpha$-particle reactions with ambient ${ }^{12} \mathrm{C}$ and ${ }^{16} \mathrm{O}$ (see below). We see that the predicted fluxes reach values $\simeq 10^{-5} \mathrm{~cm}^{-2} \mathrm{~s}^{-1}$ for $E_{\text {esc }} \geq$ $100 \mathrm{MeV}$ nucleon $^{-1}$.

The lines produced in a gaseous ambient medium are broadened by the recoil velocity of the excited nucleus. The full width at half maximum (FWHM) of the $4.44 \mathrm{MeV}$ from ${ }^{12} \mathrm{C}^{*}$ is $160 \mathrm{keV}$ and for the $\sim 6.2 \mathrm{MeV}$ from ${ }^{16} \mathrm{O}^{*}$ is $180 \mathrm{keV}$.

\section{Conclusion}

Our predicted fluxes are below currently available sensitivity limits of INTEGRAL. After $\sim 3$ Ms of exposure of the GC region, the SPI sensitivity for detection of a narrow line at energy $\sim 5$ $\mathrm{MeV}$ was $3.2 \times 10^{-5} \mathrm{~cm}^{-2} \mathrm{~s}^{-1}$. But for a broad line of $\sim 200 \mathrm{keV}$ FWHM the sensitivity limit increases by a factor of $\sim 8$. Thus it is unlikely that nuclear lines mentioned above can be detected with SPI. But future gamma-ray missions may be able to test our predictions. Thus, the GRIPS mission proposed for ESA's "Cosmic Vision" program could achieve after 5 years in orbit more than an order of magnitude sensitivity improvement over COMPTEL (in 9 years), which would allow a clear detection of the predicted gamma-ray line emission at $4.44 \mathrm{MeV}$ and $6.2 \mathrm{MeV}$ from the GC region.

We note that nuclear interactions of subrelativistic ions with ambient material can also synthesize nuclear radioisotopes, whose decay can inject positrons into the GC region. From the radioisotope production yields [12], we estimate that for $E_{\text {esc }} \sim 100 \mathrm{MeV}$ the number of positrons produced by this mechanism is $<5$ times the number of gamma-rays emitted in the 4.44 and 6.2 $\mathrm{MeV}$ lines. This limit is more than an order of magnitude lower than the positron annihilation rate measured with INTEGRAL/SPI. 


\section{Acknowledgments}

DOC and VAD are partly supported by the RFBR grant 08-02-00170-a, the NSC-RFBR Joint Research Project RP09N04 and 09-02-92000-HHC-a and by the grant of a President of the Russian Federation "Scientific School of Academician V.L.Ginzburg". KSC is supported by a GRF grant of Hong Kong Government. CMK is supported by the Taiwan National Science Council grants NSC 96-2112-M-008-014-MY3 and NSC 98-2923-M-008-001-MY3. WHI is supported by the Taiwan National Science Council grants NSC 96-2752-M-008-011-PAE and NSC 96-2111-M-008-010.

\section{References}

[1] Alexander, T. 2005, PhR, 419, 65

[2] Ayal, S., Livio, M., and Piran, T. 2000, ApJ, 545, 772

[3] Baganoff, F. K., Maeda, Y., Morris, M. et al. 2003, ApJ, 591, 891

[4] Berezinskii, V. S., Bulanov, S. V., Dogiel, V. A., Ginzburg, V. L., and Ptuskin, V. S. 1990, Astrophysics of Cosmic Rays, ed. V.L.Ginzburg, (Norht-Holland, Amsterdam)

[5] Cunha, K., Sellgren, K., Smith, V. V., Ramirez, S. V., Blum, R. D., and Terndrup, D. M. 2007, ApJ, 669,1011

[6] Donley, J. L., Brandt, W. N., Eracleous, M., and Boller, Th. 2002, ApJ, 124, 1308

[7] Gillessen, S., Eisenhauer, F., Trippe, S., Alexander, T., Genzel, R., Martins, F., and Ott, T. 2008, A Giant Step: from Milli- to Micro-arcsecond Astrometry, Proc.IAU, IAU Symposium, 248, 466; astro-ph/0810.4674

[8] Grönewegen, M. A. T., Udalski, A., and Bono, G. 2008, A\&A, 481, 441

[9] Koyama K., Maeda Y., Sonobe T. et al. 1996, PASJ, 48, 249

[10] Koyama, K., Hyodo, Y., Inui, T. et al. 2007, PASJ, 59, 245

[11] Koyama, K., Takikawa, Y., Hyodo, Y. et al. 2008, arXiv:0804.3309

[12] Kozlovsky, B., Murphy, R. J., and Ramaty, R. 2002, ApJS, 141, 523

[13] Lodders, K. 2003, ApJ, 591, 1220

[14] Muno, M. P., Baganoff, F. K., Bautz, M. W. et al. 2004, ApJ, 613, 326

[15] Phinney, E. S. 1989, Nature, 340, 595

[16] Porquet, D., Grosso, N., Predehl, P. et al. 2008, A\&A, 488, 549

[17] Rees, M. J. 1988, Nature, 333, 523

[18] Sirota, V. A., Il'in, A. S., Zybin, K. P., and Gurevich, A. V. 2005, JETP, 100, 294

[19] Sunyaev, R. A., Markevitch, M., Pavlinsky, M. 1993, ApJ, 407, 606

[20] Syer, D., and Ulmer, A. 1999, MNRAS, 306, 35

[21] Ulmer, A. 1999, ApJ, 514, 180

[22] Yuasa, T., Tamura, K., Nakazawa, K., Kokubun, M., Makishima, K., Bamba, A., Maeda, Y., Takahashi, T., Ebisawa, K., Senda, A., Hyodo, Y., Tsuru, T. G., Koyama, K., Yamauchi, S., \& Takahashi, H. 2008, PASJ, 60, S207

[23] Yusef-Zadeh, F., Roberts, D. A., Goss, W. M., Frail, D. A., and Green, A. J. 1996, ApJL, 466, 25 